\title{
Prompt GRB Optical Follow-up Experiments
}

H.S. Park, G. Williams, E. Ables, D. Band, S. Barthelmy, R. Bionta, T. Cline, N. Gehrels, D. Hartmann, K. Hurley, M. Kippen, R. Nemiroff, W. Pereira, and R. Porrata

This article was submitted to $1^{\text {st }}$ KIAS Astrophysics Workshop-Explosive Phenomena in Astrophysical Compact Objects, Seoul, Korea, May $24-27,2000$

\section{November 13, 2000}

\section{U.S. Department of Energy}

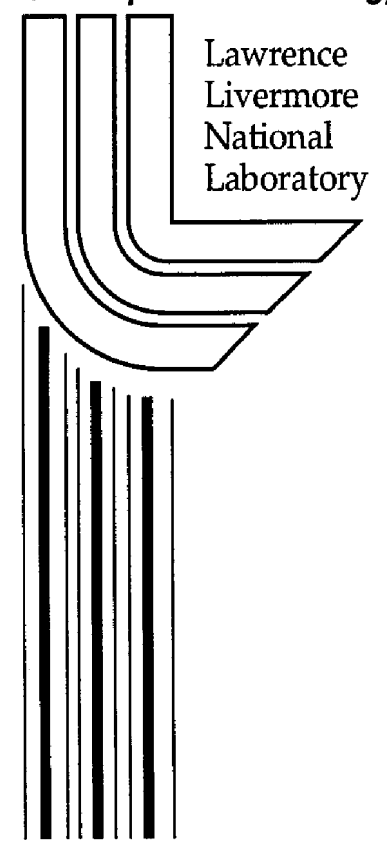




\section{DISCLAIMER}

This document was prepared as an account of work sponsored by an agency of the United States Government. Neither the United States Government nor the University of California nor any of their employees, makes any warranty, express or implied, or assumes any legal liability or responsibility for the accuracy, completeness, or usefulness of any information, apparatus, product, or process disclosed, or represents that its use would not infringe privately owned rights. Reference herein to any specific commercial product, process, or service by trade name, trademark, manufacturer, or otherwise, does not necessarily constitute or imply its endorsement, recommendation, or favoring by the United States Government or the University of California. The views and opinions of authors expressed herein do not necessarily state or reflect those of the United States Government or the University of California, and shall not be used for advertising or product endorsement purposes.

This is a preprint of a paper intended for publication in a journal or proceedings. Since changes may be made before publication, this preprint is made available with the understanding that it will not be cited or reproduced without the permission of the author.

This report has been reproduced

directly from the best available copy.

Available to DOE and DOE contractors from the

Office of Scientific and Technical Information

P.O. Box 62, Oak Ridge, TN 37831

Prices available from (423) 576-8401

http://apollo.osti.gov/bridge/

Available to the public from the

National Technical Information Service

U.S. Department of Commerce 5285 Port Royal Rd., Springfield, VA 22161

http://www.ntis.gov/

OR

Lawrence Livermore National Laboratory

Technical Information Department's Digital Library

http://www.llnl.gov/tid/Library.html 


\title{
Prompt GRB Optical Follow-up Experiments
}

\author{
H. S. Park ${ }^{1}$, G. Williams ${ }^{8}$, E. Ables ${ }^{1}$, D. Band ${ }^{5}$, S. Barthelmy ${ }^{3}$, R.

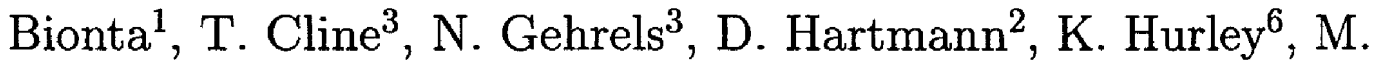

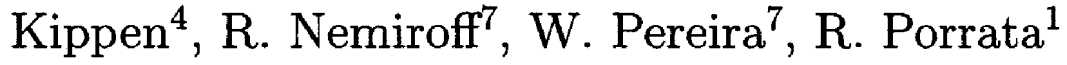 \\ ${ }^{1}$ Lawrence Livermore National Laboratory, Livermore, CA 94550 \\ ${ }^{2}$ Dept. of Physics and Astronomy, Clemson University, Clemson, SC 29634-1911 \\ ${ }^{3}$ NASA/Goddard Space Flight Center, Greenbelt, MD 20771 \\ ${ }^{4}$ NASA/Marshall Space Flight Center, Huntsville, AL 35812 \\ ${ }^{5}$ Los Alamos National Laboratory, Los Alamos, NM 87545 \\ ${ }^{6}$ Space Sciences Laboratory, University of California, Berkeley, CA 94720-7450 \\ ${ }^{7}$ Dept. of Physics, Michgan Technological University, Houghton, MI 49931 \\ ${ }^{8}$ University of Arizona, Tucson, AZ 85721
}

\begin{abstract}
.
Gamma Ray Bursts (GRBs) are brief, randomly located, releases of gamma-ray energy from unknown celestial sources that occur almost daily. The study of GRBs has undergone a revolution in the past three years due to an international effort of follow-up observations of coordinates provided by Beppo/SAX and IPN GRB. These follow-up observations have shown that GRBs are at cosmological distances and interact with surrounding material as described by the fireball model. However, prompt optical counterparts have only been seen in one case and are therefore very rare or much dimmer than the sensitivity of the current instruments. Unlike later time afterglows, prompt optical measurements would provide information on the GRB progenitor. LOTIS is the very first automated and dedicated telescope system that actively utilizes the GRB Coordinates Network (GCN) and it attempts to measure simultaneous optical light curve associated with GRBs. After 3 years of running, LOTIS has responded to 75 GRB triggers. The lack of any optical signal in any of the LOTIS images places numerical limits on the surrounding matter density, and other physical parameters in the environment of the GRB progenitor. This paper presents LOTIS results and describes other prompt GRB follow-up experiments including the Super-LOTIS at Kitt Peak in Arizona.
\end{abstract}

\section{INTRODUCTION}

The dramatic breakthrough in our understanding of GRBs occurred when the high resolution $\mathrm{x}$-ray detector on the Beppo/SAX satellite was able to determine the position of a GRB with sufficient accuracy [1] to enable a large telescope to 
observe a faint, fading afterglow days later. Optical [2] and radio afterglows now have been observed for about a dozen GRBs during the last two years. These longlasting but faint afterglows have been successfully explained in the fireball models as the result of the heating up of surrounding material by the GRB energy release [3]. From the spectra of the apparent hosts of the afterglows we now know that the GRBs are at cosmological distances and also have gained some understanding of the GRB energy output, ambient environment, and dynamics.

The goal of the LOTIS experiment is to measure prompt visible emission occurring within seconds of the gamma-ray energy release and presumably containing information about the GRB progenitor. To accomplish this we developed and have been operating an automated wide field-of-view telescope for over 3 years at Lawrence Livermore National Laboratory (LLNL) that responds to triggers distributed by the Gamma-ray burst Coordinate Distribution Network (GCN) by rapidly imaging the GRB coordinate error boxes. This instrument collected data on over 75 GRBs and in all cases found no evidence for prompt visible emission down to its sensitivity limit.

During the onset of the rainy season in Livermore, a similar competing instrument, ROTSE, at Los Alamos, NM, responded to a GCN trigger from GRB990123 whose GRB error box was later localized by the Beppo/SAX detection of a fading $\mathrm{X}$-ray source which led to the later detection of optical afterglows [4]. Subsequent analysis of the ROTSE images showed a prompt optical transient at mag 9 [5]. A signal this bright would clearly have been seen by LOTIS, however to this date prompt optical signals have not been seen from any other GRB in the large LOTIS sample. This burst was a very strong event having a peak gamma-ray flux of $16.4 \gamma$ $\mathrm{cm}^{-2} \mathrm{~s}^{-1}$ and a total fluence of $2.0 \times 10^{-4} \mathrm{erg} \mathrm{cm}^{-2}$. In addition, GRB990123 exhibited an extremely hard gamma-ray spectrum and the prompt optical flux measurement is not consistent with an extrapolation of the burst spectrum to low energies [6]. With only this single case of bright prompt optical emission, it is difficult to determine which burst characteristics might predict such a flash. We attempt to compare the LOTIS limits with the observed prompt signal from GRB990123 to constrain the physical parameters of the reverse-shock model suggested by Sari \& Piran [7].

\section{LOTIS}

LOTIS was constructed to rapidly cover the real-time GCN trigger error box, which was limited by the BATSE $1 \sigma$ error of $2 \sim 10^{\circ}$. This large error requires wide field-of-view optics to obtain statistically meaningful results. LOTIS utilizes commercially available Canon $\mathrm{f} / 1.8$ telephoto lenses with $200 \mathrm{~mm}$ focal lengths yielding effective apertures of $110 \mathrm{~mm}$ diameter. The electronic focal plane sensors are $2048 \times 2048$ pixel Loral 442 A CCDs with $15 \times 15 \mu$ m pixels driven by custom read-out electronics. Each Canon telephoto lens/camera assembly has a field-ofview of $8.8 \times 8.8^{\circ}$ with a pixel scale of $15^{\prime \prime}$. Four cameras are arranged in a $2 \times 2$ 

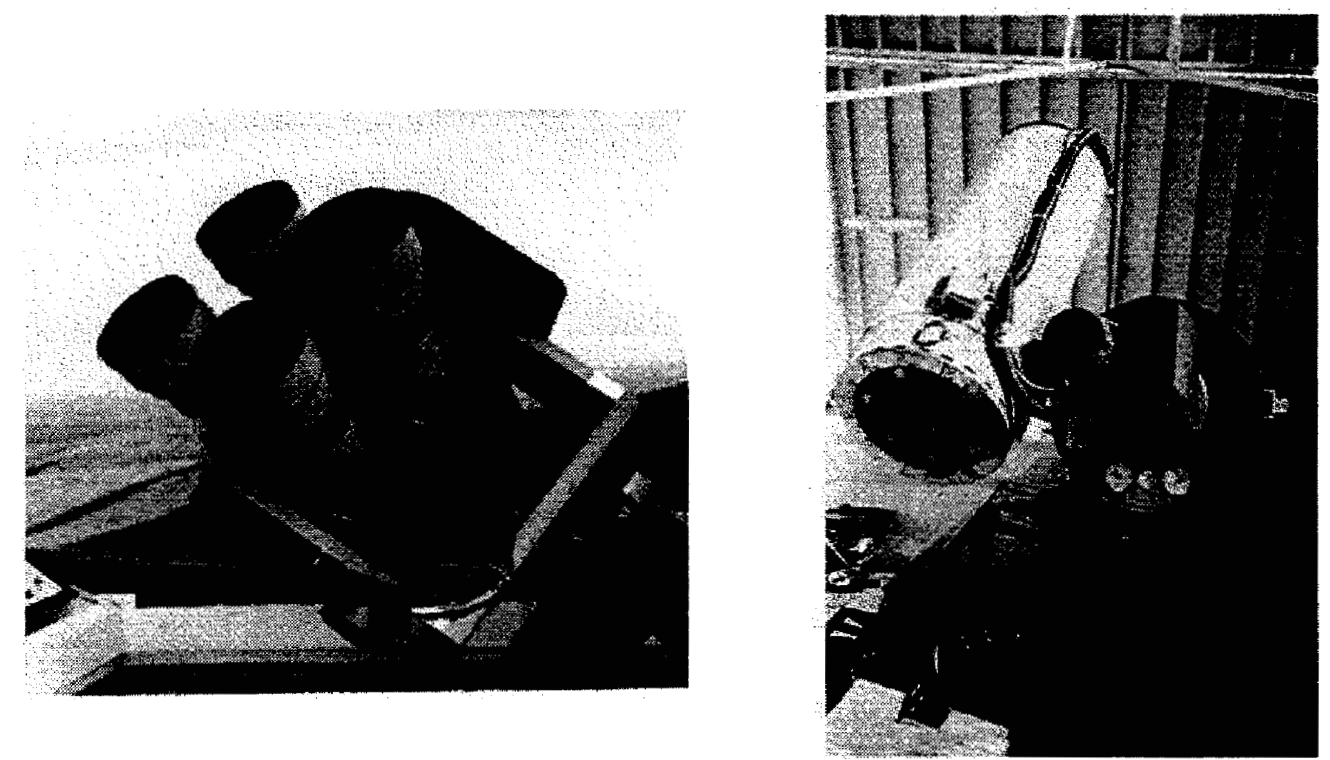

FIGURE 1. Lotis and Super-LOTIS telescopes.

array to cover a total field-of-view of $17.4 \times 17.4^{\circ}$ overlapping $0.2^{\circ}$ in each dimension. LOTIS is located 25 miles east of Livermore, CA. The first picture of Figure 1 shows the LOTIS system.

\section{LOTIS RESULTS \& INTERPRETATION}

During more than 1330 possible nights of observations (Oct. 1996 through May 2000 ), LOTIS responded to $145 \mathrm{GCN}$ triggers. Of these, 75 triggers were unique GRB events. The quality of the LOTIS coverage for a given event depended on: the observing conditions, the LOTIS response time, the error in the rapidly distributed GRB coordinate, the size of the final error box, and the duration of the GRB. The following table lists the 13 events for which LOTIS achieved the best overall coverage. These results have been published [8], [9], [10].

Table 1 shows our best events during this period. Since LOTIS did not see any optical activity in the visible, correlated with any of these events, we determine from the conditions in each case an upper limit on the brightness of any possible visible signal missed by LOTIS.

This set of LOTIS upper limits has been used to determine if typical GRBs, for which LOTIS has shown not to have counterparts, are similar to GRB990123, the only GRB event ever observed to have a prompt visible counterpart. Since GRB990123 also had an unusually strong gamma-ray emission, it is interesting to compare the LOTIS limits with predicted optical signals based on the measured optical intensity of GRB990123 scaled by the ratio of the x-ray intensity for each LOTIS burst to the x-ray intensity of GRB990123. Since the x-ray intensity of a 
TABLE 1. Best LOTIS events and scaled GRB990123 limits

\begin{tabular}{lccccccc}
\hline Event & $\begin{array}{c}\text { Peak } \\
\text { Flux }\end{array}$ & Fluence & $\begin{array}{c}\text { Burst } \\
\text { duration }\end{array}$ & $\begin{array}{c}\text { LOTIS } \\
\text { response } \\
\text { time }\end{array}$ & $\begin{array}{c}\text { LOTIS } \\
\text { limit }\end{array}$ & $\begin{array}{c}\text { Scaled } \\
\text { mag to } \\
\text { peak } \\
\text { flux }\end{array}$ & $\begin{array}{c}\text { Scaled } \\
\text { mag to } \\
\text { fluence }\end{array}$ \\
\hline 961017 & 1.98 & 5.07 & 1.2 & 11.0 & 11.5 & 11.3 & 15.9 \\
961220 & 1.60 & 18.1 & 9.8 & 9.0 & 11.5 & 11.5 & 14.5 \\
970223 & 16.84 & 968 & 16.3 & 11.5 & 11.0 & 9.0 & 10.2 \\
970714 & 1.32 & 17.1 & 2.0 & 14.1 & 11.3 & 11.7 & 14.6 \\
970919 & 0.77 & 22.5 & 20.9 & 11.8 & 11.5 & 12.3 & 14.3 \\
971006 & 1.79 & 258 & 48.1 & 17.1 & 12.1 & 11.4 & 11.7 \\
971227 & 2.11 & 9.25 & 6.8 & 10.0 & 12.3 & 11.2 & 15.3 \\
990129 & 4.99 & 585 & 200 & 140.8 & 14.5 & 10.3 & 10.8 \\
990308 & 1.26 & 164 & 50 & 132.1 & 13.5 & 11.8 & 12.2 \\
990316 & 3.67 & 529 & 40 & 13.6 & 14.3 & 10.6 & 10.9 \\
990413 & 2.57 & 68.1 & 15 & 13.0 & 14.0 & 11.0 & 13.1 \\
990803 & 12.19 & 1230 & 28 & 15.0 & 14.5 & 9.3 & 10.0 \\
990918 & 3.17 & 25.2 & 6.5 & 8.3 & 14.3 & 10.8 & 14.2 \\
\hline
\end{tabular}

burst can be quantified by its peak flux and/or its total fluence we present predictions for both in the last 2 columns of Table 1 . When we compare these results with the LOTIS limiting magnitude in the column 5 , we find that the predicted prompt optical signals are in most cases brighter that the LOTIS upper limits (especially after GRB 970919 when LOTIS was upgraded). This suggests that GRB990123 was not a typical GRB.
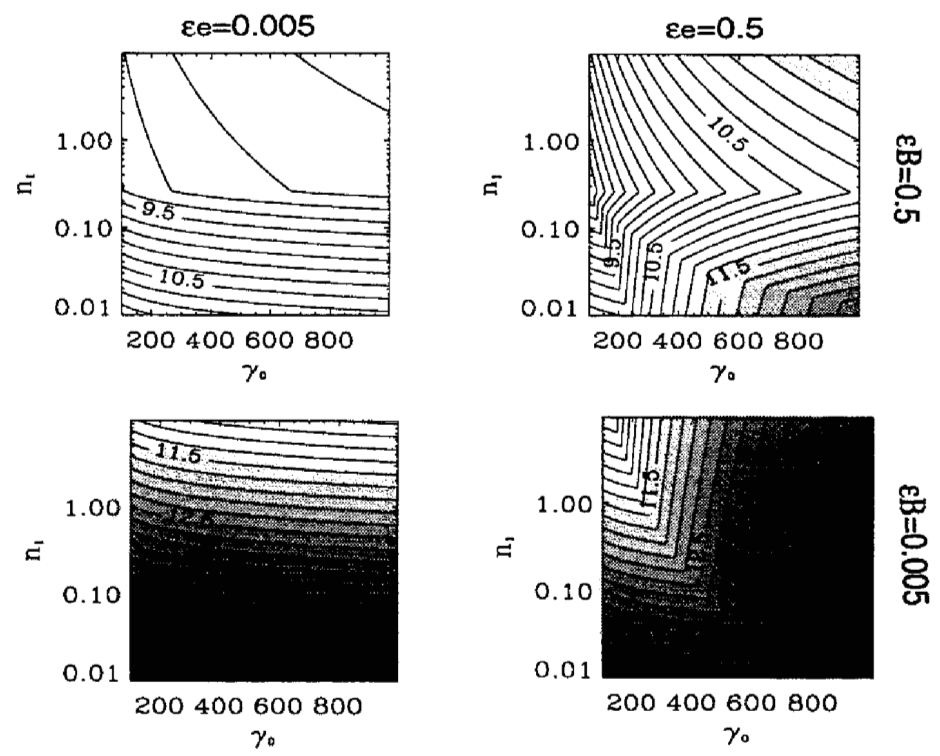

FIGURE 2. LOTIS constraints on GRB production parameters. The darker regions are preferred by LOTIS. 
The LOTIS results have also been used by Sari \& Piran to constrain their external-reverse-shock GRB model. This model assumes that the fraction of the GRB energy emitted in the optical band depends on the values of the cooling frequency and the characteristic synchrotron frequency. Extending this theory to the external reverse shock model for prompt optical signal, requires physical parameters of $\varepsilon_{e}$ and $\varepsilon_{B}$ that are the fraction of equipartition energy in the electrons and magnetic field; $\mathrm{n}$, the density of the surrounding matter in $\mathrm{cm}^{-3}$; and $\gamma_{o}$ is the initial Lorentz factor. Assuming that the observable parameters of the total fluence and the observation time are fixed at $\mathrm{S}=2.33 \times 10^{-7} \mathrm{erg} \mathrm{cm}^{-2}$ and $\mathrm{t}=10 \mathrm{~s}$, we predict the likely gamma-ray burst production environment shown as the contour lines are shown in Figure 2. The darker regions in these plots are favored by LOTIS limits. From this analysis LOTIS results imply that the GRBs are created at low surrounding matter density, high initial Lorentz factors, low equipartition energy in the magnetic field, and high equipartition energy in the electron.

\section{LOTIS SKY PATROL DATA}

The typical rate for burst triggers is $\sim 1 /$ month. While not responding to burst triggers, LOTIS systematically acquires all sky data $\sim 4$ times/night. We have just begun to analyze this data set for variable objects. Figure 3 shows the light curves for two example stars over 3 months selected from the field near SS-Cyg. This data set includes most seeing conditions experienced over the 3 month period including periods of bright Moon phase. We analyzed the data utilizing the daophot photometry code to produce the light curves. Shown in the figure 3 are the light curves for a non-varying star and a cataclysmic variable, SS-Cyg. The data for the non-varying star shows that we can monitor variability with a photometric accuracy of mag 0.03 for mag 12.0 stars. The second panel shows the light curve for SS-Cyg. We can clearly monitor its outbursts. When the outburst was brighter than mag 9.5, SS-Cyg saturated the camera. Encouraged by this result, we plan to expand this analysis to all of the data.

\section{SUPER-LOTIS}

Super-LOTIS is a next-generation system designed to be sensitive enough to detect GRB prompt optical levels predicted by current theories. The telescope (second picture in Figure 1) is a Boller and Chivens $0.6 \mathrm{~m}$ reflective telescope of $\mathrm{f} / 3.5$. We automated the telescope for GRB follow-up work by adding computer controllable drives. These drives can point to any part of the sky within $30 \mathrm{~s}$ upon receipt of a GCN trigger. We also designed and fabricated a custom 4-element coma corrector to match the point spread function to the pixel scale at the corners of the imaging CCD. The sensor is an upgraded LOTIS CCD camera. Super-LOTIS has a $0.84 \times 0.84^{\circ}$ field-of-view $\left(1.5^{\prime \prime} /\right.$ pixel $)$ sufficient to cover the error boxes expected from upcoming GRB satellite missions. 

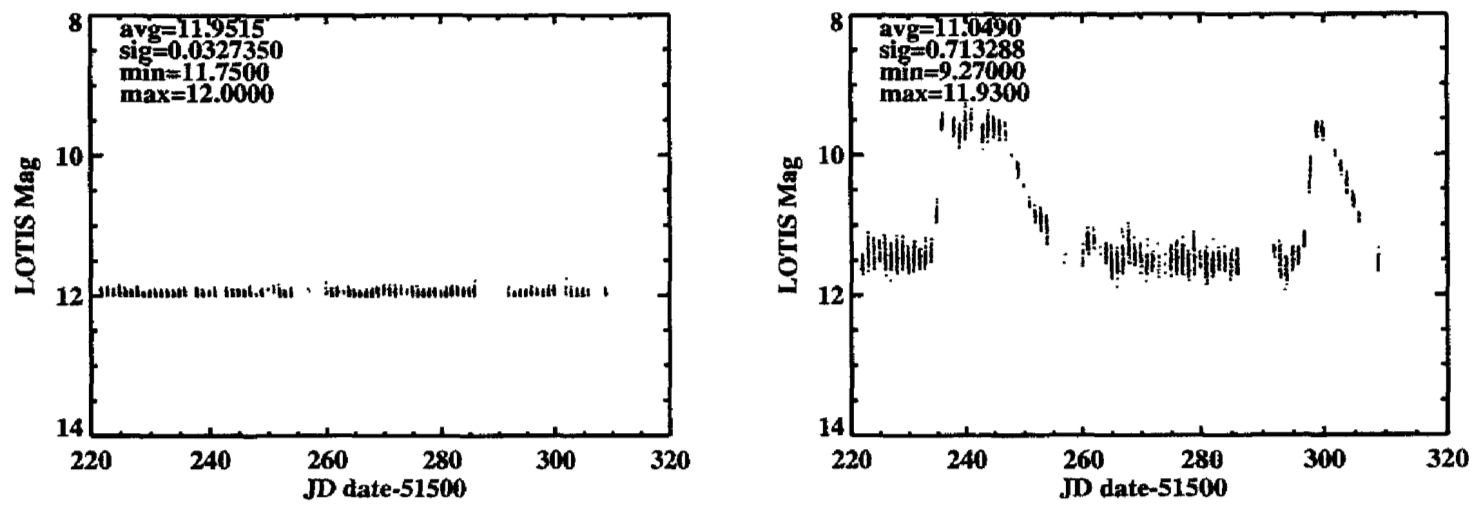

FIGURE 3. LOTIS sky patrol data: light curves for a non-varying star and a cataclysmic variable, SS-Cyg

Our data acquisition system includes custom readout electronics, a custom hardware power control unit, a weather station and a housing control unit. Extensive on-line scheduling software has been written to handle various triggers. Priority is given to the most recent trigger that has smallest error box. Our scanning strategy and automation allows us to record GRB optical activity as early as $30 \mathrm{~s}$.

In April 2000, we moved Super-LOTIS to the Kitt Peak National Observatory and are ready to receive real-time GRB coordinates form the HETE-II satellite which was successfully launched in October, 2000.

\section{OTHER PROMPT FOLLOW-UP EXPERIMENTS}

TAROT: TAROT-1 has been operating since July 1999 . It uses $20 \mathrm{~cm}$ aperture, $\mathrm{f} / 3.2$ telescope with a $1024 \times 1270$ pixel CCD. Its field-of-view is $2 \times 2^{\circ}$ and it is located in France. Because of its small-field-of-view optics, it didn't cover many real-time BATSE triggers [11] even though its mount can respond to a burst trigger within a few seconds. They have a plan to construct TAROT-2 that has bigger optics ( $1.5 \mathrm{~m}$ aperture) and a $2000 \times 2000 \mathrm{CCD}$ with a spectrometer. This system is under development.

ROTSE: ROTSE-I is similar to the LOTIS except the CCD array which has smaller pixel size thus the total field of view is $16.4 \times 16.4^{\circ}$, slightly smaller than LOTIS. The ROTSE collaboration is now constructing ROTSE-II which has $45 \mathrm{~cm}$ aperture with $\mathrm{f} / 1.9$. This system is under construction as of September, 2000 .

BOOTES: BOOTES utilizes $30 \mathrm{~cm} \mathrm{f} / 3.3$ telescope with a $1530 \times 1020$ pixel CCD. Its total field of view is $49^{\prime} \times 33^{\prime}$ and it is operating in Spain. They have responded to many BATSE triggers but again its small field-of-view provided only limited coverage of the GRB error boxes [12].

There are other telescopes under construction including YSTAR [13] that will attempt to automatically respond to burst triggers given by GRB satellites but not many are in operation as of September, 2000. 


\section{FUTURE AND CONCLUSION}

The promptly responding automatic telescopes require real-time burst coordinate triggers from GRB satellites. In June 2000, the CGRO that distributed BATSE coordinates in real-time was shut-down. Until HETE-II is fully operational, only Beppo/SAX and the IPN are distributing coordinates with a typically delay of 1 2

days. For these events, we examine the sky-patrol archives for the earliest LOTIS image taken after the GRB of the location of the Beppo/SAX or IPN coordinates. In some cases the archived images are taken as early as $2 \sim 4$ hour after the GRB.

The trigger delay problem will be solved when the HETE-II is fully operational and starts distributing coordinates in November, 2000. Other satellites such as Integral (2002) and SWIFT (2003) will provide more frequent real-time triggers as well.

Observation and measurement of prompt optical signals from GRBs will greatly enhance our understanding of the GRB progenitor. LOTIS results show that the flux of the prompt optical emission does not scale with gamma-ray peak flux or fluence. With LOTIS and Super-LOTIS, we will be able to observe GRB optical activity from $10 \mathrm{~s}$ to many hours after the event down to a magnitude $14 \sim 19$. With HETE-II and other prompt GRB coordinate distributing satellites, we will be able to measure early time optical activity.

This work was performed under the auspices of the U.S. Department of Energy by University of California Lawrence Livermore National Laboratory under contract No. W-7405-Eng-48. This work is also supported by the NASA contract number S-03975G.

\section{REFERENCES}

1. Costa, E., et al., Nature, 387, 783 (1997).

2. van Paradijs, J., et al., Nature, 386, 686 (1997).

3. Piran, T., Phy. Rep.,, 314, 575 (1999).

4. Odewahn, S., et al., GCN Circular, 201 (1999).

5. Akerlof, C., et al., Nature, 398, 400 (1999).

6. Briggs, M., et al., $A p J, \mathbf{5 2 4}, 82$ (1999).

7. Sari, R. \& Piran, T., ApJ, 517, L109 (1999).

8. Park, H., et al., ApJ, 490, L21 (1997).

9. Williams, G., et al., $A p J, \mathbf{5 1 9}$, L25 (1999).

10. Williams, G., et al., AIP Conf. Proc., 526, 250 (2000).

11. Boer, E., et al., AIP Conf. Proc. 526, 255 (2000).

12. Castro-Tirado, A., et al., AIP Conf. Proc. 526, 260 (2000).

13. Byun, Y., et al., These proceedings (2001). 